Ann. Zootech., I980, 29 (4), 387-400.

\title{
Efficacité biologique pour le poulet de chair du tourteau d'arachide traité à l'ammoniac ou à la monométhylamine en vue de l'inactivation des aflatoxines
}

\author{
J. DELORT-LAVAI, G. VIROBEN et L. P. BORGIDA $\left({ }^{*}\right)$ \\ avec la collaboration technique de \\ F. Doulay, G. Lecannu et F. de Monredon \\ Laboratoire de Technologie des Aliments des Animaux, \\ Centre de Recherches de Nantes, I.N.R.A., \\ Chemin de la Géraudière, $44^{\circ} 7^{2}$ Nantes Cédex (France)
}

\begin{abstract}
Résumé
Le tourteau d'arachide pollué par l'aflatoxine peut être efficacement décontaminé par action de l'ammoniac gazeux sous pression, en milieu peu hydraté, ou de la méthylamine à $\mathrm{pH}$ élevé. Ces traitements accroissent la teneur en azote non protéique des substrats et en réduisent la teneur en cystine.

Substitué à un même poids de tourteau témoin, le tourteau d'arachide traité à l'ammoniac n'a pas d'effet négatif sur l'ingestion alimentaire et ne réduit que de $3 \mathrm{p}$. Ioo l'indice de conversion alimentaire du régime. Dans les mêmes conditions expérimentales, l'action de la méthylamine est préjuđiciable à l'appétence; elle réduit le gain de poids des animaux et surtout diminue de 20 p. IOO l'efficacité alimentaire d'un régime à base de maïs.

En régime semi-purifié, dont le tourteau constitue la seule source protéique, l'indice de conversion alimentaire est đirectement proportionnel à la quantité đ'acides aminés soufrés réellement ingérée par le poulet de chair. A cet égard, les résultats observés avec l'ammoniac sont plus élevés qu'avec la méthylamine, en rapport direct avec l'inappétence que cette substance confère aux tourteaux traités.

A partir de ces résultats, il est possible d'établir les recommandations suivantes pour l'incorporation des tourteaux traités dans l'alimentation du poulet de chair :

- formuler le régime non sur la base du taux d'azote, dosé par la méthode de Kjeldahl dans le produit traité, mais après correction de l'azote non protéique ajouté par le traitement;

- tenir compte de la teneur réelle en cystine đu tourteau;

- ne pas dépasser une dose de 0,15 p. roo d'azote ammoniacal dans le régime du poulet de chair (contre $0,3 \mathrm{p}$. Ioo pour la poule pondeuse et $0,1 \mathrm{p}$. roo chez le porc à l'engrais), pour limiter les risques d'inappétence ou de toxicité de l'aliment traité à l'ammoniac. Des doses trois fois plus faibles dans le cas du traitement à la méthylamine sont suggérées par le présent essai.
\end{abstract}

(*) Adresse actuelle : C.O.F.N.A., 25, rue du Rempart, 37018 Tours Cédex. 


\section{1. - Introduction}

Sous forme gazeuse ou en solution, le champ d'application de l'ammoniac en alimentation animale s'étend constamment. A son effet fongistatique (MOREAU, I976) s'ajoute de fait un rôle actif d'enrichissement en azote non protéique des aliments et d'inactivation de facteurs antinutritionnels ou de contaminants des aliments des animaux. Son action est particulièrement efficace pour la destruction des aflatoxines des céréales et des tourteaux (DOLLEAR, 1969). Toutefois, dans les conditions de durée, de température et de pression d'un traitement industriel efficace, il a été observé une réduction du taux de cystine des tourteaux de coton (WALDROUP et al., I976) et d'arachide (VIROBEN et al., I978), d'ailleurs confirmée par d'autres auteurs sur le maïs (McGher et al., I979). De plus, l'enrichissement en azote non protéique qui résulte de ce traitement remet en question la validité du critère des protéines brutes $(\mathrm{N} \times 6,25)$, seul reconnu dans les transactions commerciales pour l'évaluation des tourteaux.

D'autres agents alcalins, comme la monométhylamine, assurent une détoxification efficace du tourteau d'arachide (DOLLEAR et al., I968). Plus récemment, MANN et al. (I97I) ont montré l'intérêt de l'addition de ce même réactif, avec ou sans apport de soude, sur la disparition de l'aflatoxine $B_{1}$ d'un tourteau de coton contaminé. En raison de la très forte odeur, et donc du caractère potentiellement répulsif du produit traité, GIDDEY, BRANDT et BUNTER (I977) parviennent à un résultat significatif en ramenant à 0,5 p. Ioo le taux de monométhylamine ajoutée, en présence d'hydroxyde de calcium à température élevée et avec addition d'une faible quantité d'eaul. Cette substance a, sur la composition et les caractéristiques physico-chimiques de la fraction azotée des tourteaux, un effet similaire à celui de 1'ammoniac.

C'est pourquoi, il est apparu souhaitable, dans le présent essai, de comparer l'efficacité biologique pour le poulet de chair, de tourteaux d'arachide contaminés par l'aflatoxine à celle de produits détoxifiés, afin d'examiner l'influence des traitements proposés, en s'attachant particulièrement à rechercher si ces derniers exerçaient un effet sur la disponibilité biologique des acides aminés soufrés, eu égard à la destruction partielle de cystine observée in vitro.

\section{2. - Matériel et méthodes}

L'évaluation des traitements de détoxification des tourteaux a fait l'objet de deux essais séparés, non directement comparables entre eux. L'effet de l'ammoniac a été étudié chez le poulet de chair entre 3 et 7 semaines à une température proche de l'ambiante; celui de la méthylamine a pu être réalisé entre Io et 24 jours dans les nouvelles installations climatisées désormais disponibles au Centre de Recherches de Nantes.

\section{I. - Traitements à l'ammoniac}

\section{II. - Tourteaux expérimentaux}

Le traitement de tourteaux d'arachide par l'ammoniac en vue de l'inactivation de l'aflatoxine a été décrit dans un mémoire antérieur (VIROBEN et al., I978). Deux tourteaux $\mathrm{AO}$ et $\mathrm{AO}_{1}$, contenant respectivement 0,98 et I,I4 p.p.m. 
TABLEAU I

Quelques caractéristiques physico-chimiques de tourteaux d'arachide traités à l'ammoniac (série $A$ ) ou à la monométhylamine (série $B$ )

Some physico-chemical parameters of $\mathrm{NH}_{3}$ (série A) or MMA (série B) treated peanut meals

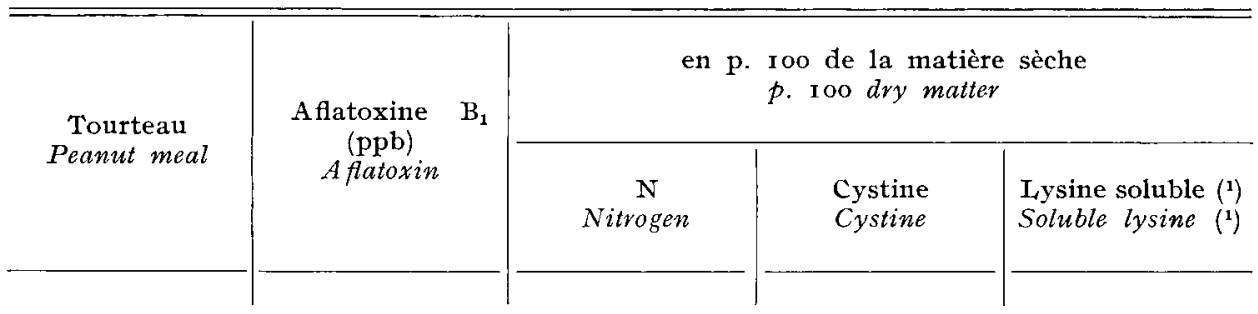

a) Traitement à l'ammoniac

Ammonia treatment

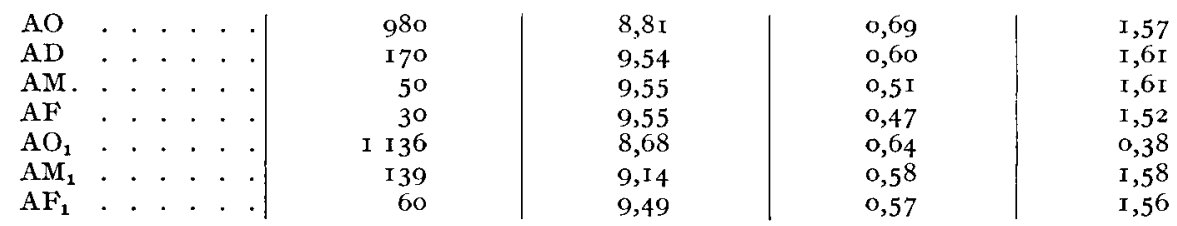

b) Traitement à la monométhylamine MMA treatment

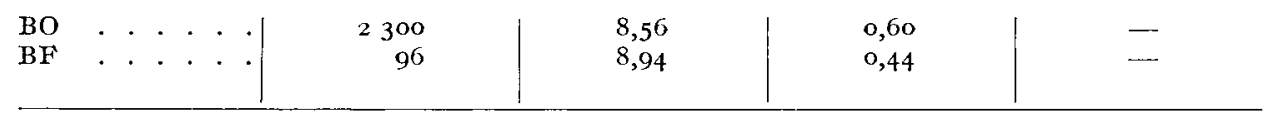

(I) Résultats d'une hydrolyse enzymatique des protéines et d'un dosage microbiologique par $L$. mesenteroides (After enzymatic proteolysis and microbiological determination with L. mesenteroides).

d'aflatoxine $B_{1}$, ont subi l'action de l'ammoniac sous des pressions de $\mathrm{I}$ bar (traitement doux $A D$ ), 2 bars (traitement moyen $A M$ et $A_{1}$ ) ou 3 bars (traitement fort $\mathrm{AF}$ ou $\mathrm{AF}_{1}$ ). Ils sont comparés, dans le présent essai d'évaluation nutritionnelle, à un tourteau d'arachide $T$ à faible teneur en aflatoxine $(0,056$ p.p.m.). Quelques caractéristiques majeures de ces tourteaux, tirées de la précédente étude (VIROBEN et al., I978), sont rappelées dans le tableau I.

\section{2.r2. - Animaux et protocole expérimental}

Cent cinquante coquelets d'un jour, bagués et vaccinés à la naissance, sont élevés au sol à une température de $28-30^{\circ} \mathrm{C}$. Ils reçoivent durant 3 semaines un aliment commercial de "démarrage", en farine jusqu'au $\mathrm{I}_{3} \mathrm{e}$ jour, en granulés de $2,5 \mathrm{~mm}$ par la suite. A partir du quinzième jour, les animaux sont adaptés aux cages individuelles, à température ambiante $\left(22 \pm 2^{\circ} \mathrm{C}\right)$ et à un éclairement de I4 heures par jour. Ils sont répartis en lots homogènes sur la base de leur poids au $2 \mathrm{I}$ jour et de leur gain de poids depuis le $5^{\mathrm{e}}$ jour et affectés aux traitements expéri- 
mentaux dans les 72 cages individuelles de la batterie, où le poids et la consommation alimentaire sont enregistrés chaque semaine entre 3 et 7 semaines d'âge. Les pesées sont toujours effectuées après un jeûne hydrique de I 6 heures; la matière sèche des aliments et des refus est mesurée chaque semaine pour chaque régime.

\subsection{3. - Aliments}

Dans un premier essai, les tourteaux expérimentaux fournissent environ 60 p. Ioo de l'apport azoté, en complément du maïs qui constitue 65,2 p. Ioo des régimes isoazotés à 22 p. Ioo de protéines brutes par rapport à la matière sèche. Le taux azoté des tourteaux étant variable en fonction de l'intensité du traitement à l'ammoniac (tabl. I), la part de l'azote non protéique des régimes est ajustée par addition d'urée $(0-0,52 \mathrm{p}$. I0o), aux dépens de l'amidon de maïs $(4,2-5,3 \mathrm{p}$. I00). Des acides aminés (0,4 p. Ioo de L-lysine-monochlorhydrate et $0,1 \mathrm{p}$. Ioo de L-thréonine) sont ajoutés, ainsi qu'un complément minéral et vitaminique. La composition du complément minéral incorporé au régime à raison de $4 \mathrm{p}$. IOO, a été décrite dans un mémoire antérieur (Szylit' et al., I974); celle du complément vitaminique fournit, en $\mathrm{g}$ par Ioo $\mathrm{kg}$ d'aliments et sur support d'amidon de maïs : rétinol 0,375 ; cholécalciféro1 0,005 ; tocophérol $\mathrm{I}, 75 ; 2$-méthyl-I.4-naphtoquinone 0,69 ; thiamine $I, 2$; riboflavine 2,4 ; pyridoxine 1,76 ; acide nicotinique 20 ; acide folique 0,6 ; acide ascorbique 32 ; acide p. aminobenzoïque 28 ; inositol r oo; biotine 0,08 ; pantothénate $\mathrm{Ca} 7$; choline $\mathrm{I}_{50}$.

Pour tenir compte de la variation de teneur en azote et en cystine des tourteaux et en mesurer l'influence sur l'efficacité biologique des produits traités, un essai de supplémentation par trois taux de méthionine a été effectué sur le témoin $\mathrm{AO}$ et le produit le plus fortement traité $\mathrm{AF}$. Dans un régime dont il constituait la seule source protéique, le tourteau traité a été incorporé, soit au même taux pondéral que le témoin, soit de manière à fournir un taux azoté identique. Tous les régimes ont été supplémentés en lysine, thréonine, valine et isoleucine, en vue de répondre aux besoins du poulet de chair (Anonyme, I972); leur composition est reprise au tableau 2 . Les gains de poids et consommations d'aliments ont été enregistrés chaque semaine, les animaux étant pesés après un jeûne hydrique de I 6 heures, deux jours consécutifs au début $\left(2 \mathrm{I}^{\mathrm{e}}\right.$ et $\left.\mathrm{z} 2^{\mathrm{e}} \mathrm{i}\right)$ et à $1 \mathrm{a}$ fin $\left(49^{\mathrm{e}}\right.$ et $5^{\mathrm{e}} \mathrm{j}$ ) de l'essai. Le gain de poids est estimé par régression linéaire durant cette période; on calcule l'indice de conversion alimentaire en rapportant le gain à la consommation individuelle de matière sèche durant la période considérée.

\section{2. - Traitement par la monométhylamine}

\subsection{1. - Tourteaux expérimentaux}

Deux tourteaux d'arachide, témoin (BO) ou traité à la monométhylamine (BM) selon l'un des procédés décrits par GIDDE y et al. (I977), dosent respectivement, d'après les méthodes décrites par Viroben et al. (I978) en p. roo de la matière sèche : $\mathrm{N}: 8,56$ et 8,$94 ; \mathrm{N}$ soluble dans l'acide trichloracétique : 0,58 et 1,$03 ; \mathrm{N}$ soluble à $\mathrm{pH} 7,5: 3,82$ et $2, \mathrm{I} 0$; bases azotées volatiles, exprimées en ammoniac : 0,06 et 0,22 ; cystine : 0,60 et 0,44 ; lysine : I,50 et I,4I; tyrosine : I,36 et I,70; les autres acides aminés ne sont pas modifiés. 

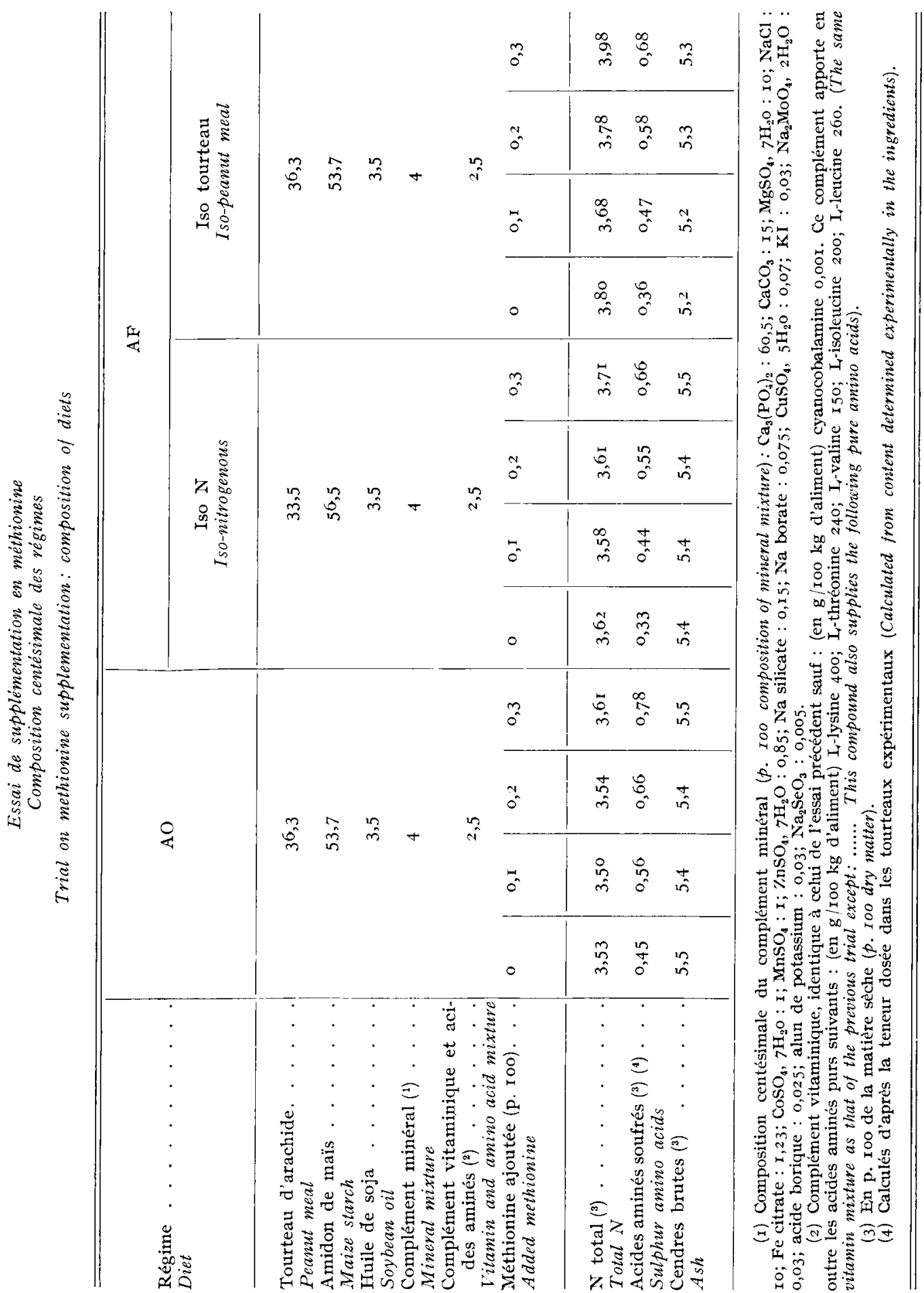


\subsection{2. - Animaux}

Ces deux produits ont été soumis à deux tests biologiques sur poulets de chair, dans les conditions suivantes :

- Le test d'efficacité globale est effectué sur 2 lots de I6 poussins HUBBard femelles placés en cages individuelles entre ro et 24 jours, après sélection des animaux sur la base du poids à ro jours et du gain de poids entre 3 et ro jours. Ils sont pesés chaque semaine après un jeûne hydrique de I 8 heures. La température

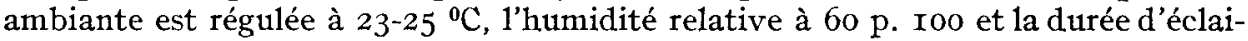
rement à I4 heures.

- L'essai de supplémentation est effectué, dans les mêmes conditions, sur 8 lots de 8 animaux.

\subsection{3. - Aliments}

Après la période de démarrage durant laquelle les animaux reçoivent un aliment commercial, les régimes expérimentaux suivants leur sont distribués en agglomérés de 2,5 $\mathrm{mm}$ :

- pour l'essai d'efficacité globale : tourteau d'arachide 24; maïs 65; L-1ysinemonochlorhydrate 0,6 ; L-thréonine $0, \mathrm{I}$; composé minéral 4 et vitaminique I; amidon de maïs 5,3 ;

- pour 1'essai de supplémentation en méthionine : tourteau d'arachide 36; L-1ysine-monochlorhydrate 0,5 et tous les autres composants comme indiqué au tableau 2 .

Les composés minéraux et vitaminiques de ces deux essais sont ceux décrits au tableau 2, avec suppression des acides ascorbique et para-aminobenzoïque et de l'inositol.

\section{3. - Résultats}

Les résultats de croissance, de consommation et d'efficacité alimentaire des régimes à base d'arachide témoin ou traité à l'ammoniac, non supplémentés en acides aminés soufrés, sont regroupés dans le tableau $3 a$. La consommation d'aliments n'est pas influencée par l'origine des tourteaux. Leur traitement et l'augmentation de teneur en azote ammoniacal qui en résulte n'ont pas d'effet négatif sur l'ingestion de matière sèche. Le gain de poids vif à partir des trois tourteaux témoins n'est pas identique : il est le plus élevé avec le tourteau AO, le plus contaminé en aflatoxine. La moindre efficacité alimentaire du témoin $\mathrm{AO}_{1}$ est à mettre en relation avec la résistance particulière de ses protéines à l'action des protéases (tabl. I). En raison du comportement particulier de ce second " témoin", l'effet $d u$ traitement ne peut être mis en évidence que sur les produits de la première série de fabrication ( $\mathrm{AO}-\mathrm{AD}-\mathrm{AM}-\mathrm{AF}$ ). Dans cette série, le traitement fort, le plus apte à ramener la teneur en aflatoxine des tourteaux fortement contaminés à moins de $5 \mathrm{p}$. roo de la teneur initiale, présente une efficacité alimentaire légèrement réduite $(-4,8 \mathrm{p}$. I00) par rapport au témoin correspondant, à même taux d'incorporation de tourteau au régime. Après traitement à la mono- 
TABLEAU 3

Test d'efficacité globale de tourteaux d'arachide témoins ou traités pour le poulet de chair Gain and FCE of control or treated peanuts meals in broilers

\begin{tabular}{|c|c|c|c|}
\hline $\begin{array}{l}\text { Tourteau d'arachide } \\
\text { Peanut meal }\end{array}$ & $\begin{array}{c}\text { Gain moyen } \\
\text { quotidien (GMQ) } \\
(\mathrm{g} / \mathrm{j}) \\
\text { Average daily gain } \\
(A D G)\end{array}$ & $\begin{array}{c}\text { Matière sèche } \\
\text { ingérée (MSI) } \\
(\mathrm{g} / \mathrm{j}) \\
\text { Dry matter intake } \\
(D M I)\end{array}$ & $\begin{array}{c}\text { Efficacité } \\
\text { alimentaire } \\
\text { (GMQ/MSI) } \\
\text { Feed conversion } \\
\text { efficiency } \\
(A D G / D M I)\end{array}$ \\
\hline
\end{tabular}

a) Traitement à l'ammoniac : résultats entre 22 et 49 jours (*) Ammonia treatment: results between 22-49 days
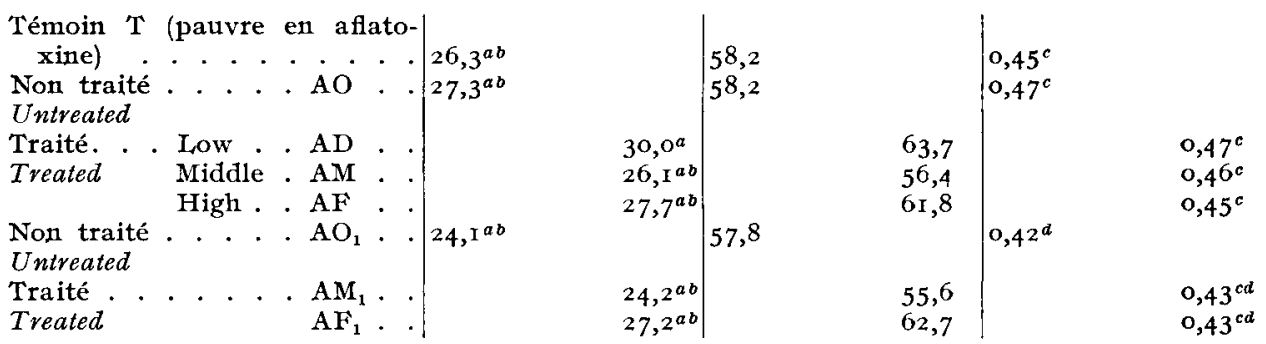

b) Traitement à la monométhylamine : résultats entre io et 24 jours $M M A$ treatment: results between I0-24 days
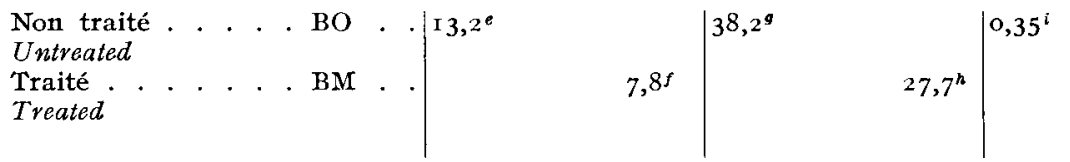

(*) Les données assorties de la même lettre ne sont pas significativement différentes (Data with the same letter do not differ significantly).

méthylamine (tabl. $3^{b}$ ), le gain de poids et l'efficacité alimentaire sont nettement réduits, sous l'effet d'une diminution de l'ingestion de matière sèche.

Dans l'essai de supplémentation en acides aminés soufrés du tourteau le plus fortement traité par l'ammoniac, l'addition de méthionine stimule l'ingestion de matière sèche et la croissance des animaux (tab1. 4a). Le gain de poids et l'efficacité alimentaire ne sont que faiblement altérés par le traitement, lorsque les régimes incluent une même proportion de tourteau; l'effet le plus marqué est la réduction de l'ingestion d'aliment contenant le tourteau traité supplémenté par I ou 2 p. I ooo de méthionine. Par contre, si l'on se fonde, pour la formulation, sur la teneur en azote ou en protéines brutes $(\mathrm{N} \times 6,25)$ du tourteau, l'effet négatif du traitement est marqué, sur tous les critères - gain de poids, matière sèche ingérée, indice de conversion alimentaire - et ce, quel que soit le niveau de supplémentation en acides aminés soufrés. Cette réduction de plus de Io p. Ioo n'est pas inattendue et correspond à l'enrichissement du tourteau en azote non protéique 


\section{TABLEAU 4}

Infuence de la supplémentation en al-methionine sur la croissance du poutet de chair et l'efficacité alimentaive d'un régime semi-purifié à base d'arachide témoin ou traité

Infuence of methionine supplement

on growth of chickens and feed conversion efficiency of semi-purified diets with control or treated peanut meal as sole source of protein

\begin{tabular}{|c|c|c|c|c|}
\hline $\begin{array}{l}\text { Tourteau } \\
\text { Peanut meal }\end{array}$ & $\begin{array}{l}\text { Méthionine } \\
\text { ajoutée } \\
\text { Added } \\
\text { methionine }\end{array}$ & $\begin{array}{c}\text { Gain moyen } \\
\text { quotidien (GMQ) } \\
(\mathrm{g} / \mathrm{j}) \\
\text { Average daily } \\
\text { gain }(A D G)\end{array}$ & $\begin{array}{c}\text { Matière sèche } \\
\text { ingérée (MSI) } \\
(\mathrm{g} / \mathrm{j}) \\
\text { Dry matter } \\
\text { intake (DMI) }\end{array}$ & $\begin{array}{c}\text { Efficacité } \\
\text { alimentaire } \\
\text { (GMQ/MSI) } \\
F C E \\
(A D G / D M I)\end{array}$ \\
\hline
\end{tabular}

a) Traitement à 1'ammoniac : résultats entre 22 et 49 jours Ammonia treatment: results between 22 and 49 days

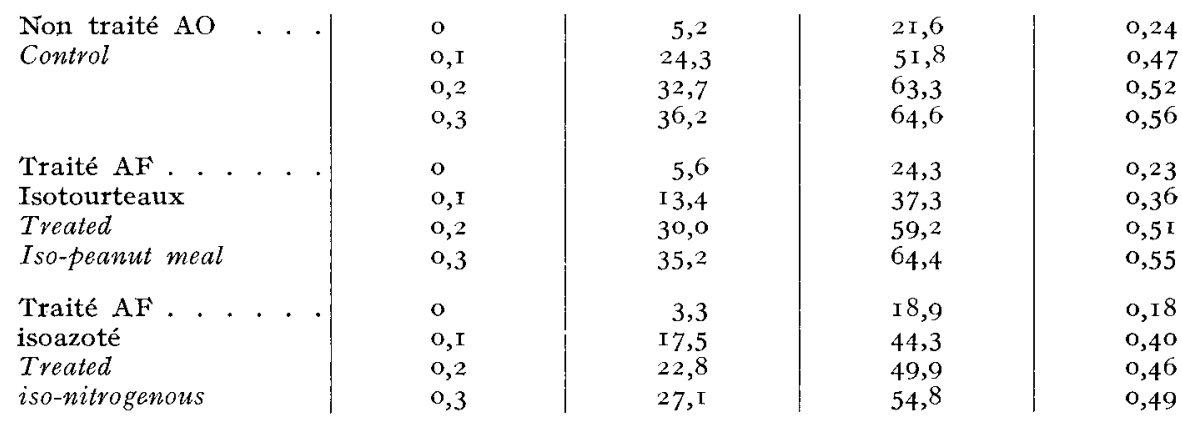

b) Traitement à la monométhylamine : résultats entre Io et 24 jours $M M A$ treatment: results between Io and 24 days

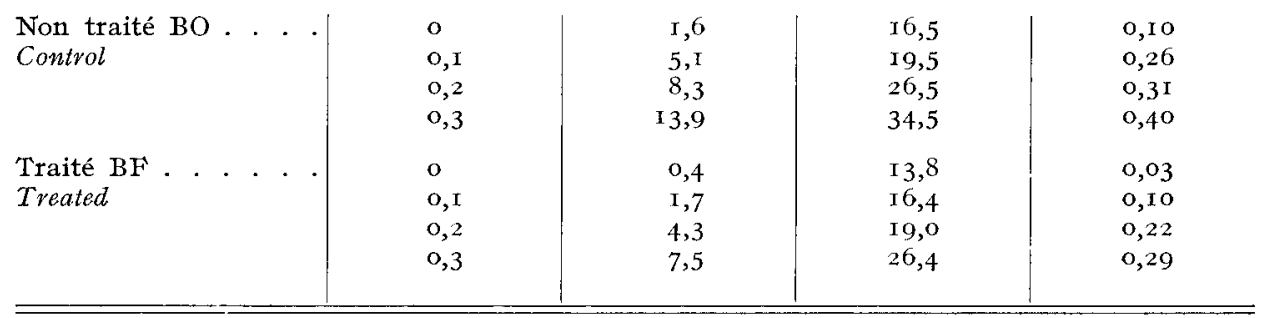

sous l'effet du traitement. En présence de monométhylamine (tabl. $4 b$ ), l'addition de méthionine à des régimes contenant une proportion de tourteau identique à celle du témoin est apparemment moins efficace, l'ingestion de tourteau traité restant toujours plus faible à chaque niveau de supplémentation en acides aminés soufrés.

Si l'on rapporte le gain de poids au taux de méthionine ajouté (fig. I $a$ et $\mathrm{I} b$ ), le produit témoin apparaît supérieur aux régimes contenant les tourteaux traités. Pour tenir compte de la variation d'ingestion liée soit à l'appétence du régime, soit 


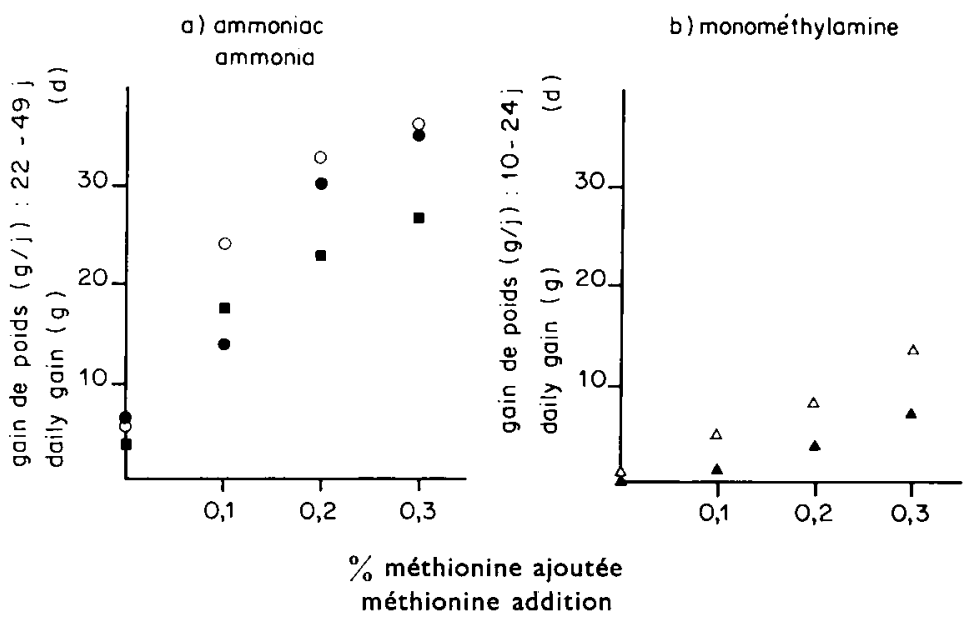

FIG. I. - Effet sur la croissance du poulet de chair du taux de supplémentation en méthionine de régimes semi-purifiés à base d'arachides traités, incorporés à même taux d'azote (回) ou de tourteau $(\bullet, \mathbf{\Delta})$ que le témoin $(0, \Delta)$.

Influence of methionine addition (in $p$. Ioo) on average daily gain ( $g$ ) of chickens receiving semipurified diets based on peanut meal; diets with the same amount of treated peanut meal $(\bullet, \mathbf{\Delta})$ or of treated peanut meal nitrogen (四) as in the control $(\mathrm{O}, \Delta)$.

à son équilibre nutritionnel, il est cependant préférable de rapporter l'indice d'efficacité alimentaire (gain/matière sèche ingérée), moins dépendant du niveau d'alimentation, à la quantité d'acides aminés soufrés réellement ingérée par chaque lot d'animaux (fig. 2). La corrélation très élevée $(r=0,984)$ entre ces deux grandeurs, malgré la différence des traitements des tourteaux et des conditions expérimentales - âge des animaux, durée de l'essai ... -, montre que la quantité d'acides aminés soufrés réellement ingérée par les animaux est le facteur déterminant de l'efficacité alimentaire du régime.

\section{Discussion}

Le test d'efficacité globale appliqué ici au tourteau d'arachide s'inspire de le " total protein efficiency " proposée par Woodнaм (I968). Cet auteur a montré que c'est à des taux azotés compris entre $I 8,5$ et $20 \mathrm{p}$. Ioo que s'extériorisent le mieux les différences d'efficacité entre les régimes où l'arachide fournit environ les $2 / 3$ de l'apport azoté, en complément d'un mélange de céréales et des acides aminés les plus limitants. A niveau d'ingestion alimentaire comparable, les différences d'efficacité alimentaire observées restent faibles, ces régimes n'étant que légèrement carencés en acides aminés soufrés.

Cette carence est notablement accrue lorsque le tourteau d'arachide est la seule source protéique alimentaire; il peut alors constituer la base de régimes servant à l'évaluation de la disponibilité de la méthionine (HARWOOD et SHRIMPToN, 1969). Comme le niveau d'ingestion influe très fortement, chez le poulet de chair, sur le gain de poids et l'efficacité alimentaire, il a été fréquemment suggéré de rapporter l'effet d'une supplémentation plutôt à l'indice de conversion ali- 
mentaire qu'au gain de poids (Gupta et al., 1958; Miller et al., I965); ce mode d'expression paraît particulièrement adapté au présent essai dans lequel 1'un des composants d'un régime est susceptible d'en altérer à la fois l'équilibre nutritionnel et l'appétence. Dans le même esprit, Combs, Bossard et ChILds (I968) ont proposé de rapporter le critère de gain ou d'efficacité non pas au taux d'incorporation de l'acide aminé limitant du régime, mais à la quantité qui en est réellement ingérée par l'animal, exprimée en échelle linéaire ou, comme le signalent MILLER et al. (I 965), en coordonnées semi-logarithmiques. Dans la présente expérience, la corrélation entre indice de conversion alimentaire et quantité d'acides aminés soufrés ingérée, en échelle logarithmique, et ce, dans des conditions expérimentales d'âge, d'origine génétique et de régime alimentaire très diverses, est élevée $(r=$ 0,983 ). Le fait que les données relatives aux tourteaux traités puissent être alignées avec celles des tourteaux témoins, signifie qu'il n'y a pas de réduction de la disponibilité de la fraction azotée et notamment des acides aminés soufrés sous l'effet des traitements et que ceux-ci n'ont d'effet sur l'animal que par la réduction du taux de cystine et par l'inappétence qu'ils confèrent aux produits détoxifiés.

L'interprétation des essais sur animaux n'est pas dépourvue d'ambiguité : la réduction de teneur en aflatoxine est en effet compensée, dans les produits traités, par l'accroissement de teneur en azote non protéique et par la réduction du taux d'acides aminés soufrés.

L'inactivation de l'aflatoxine est bien confirmée. LEE et al. (I974), CUCULLU et al. (1976) ont mis en effet en évidence les produits de sa dégradation et leur innocuité et, dans une expérience modèle, VESONDER et al. (I975) ont montré sa

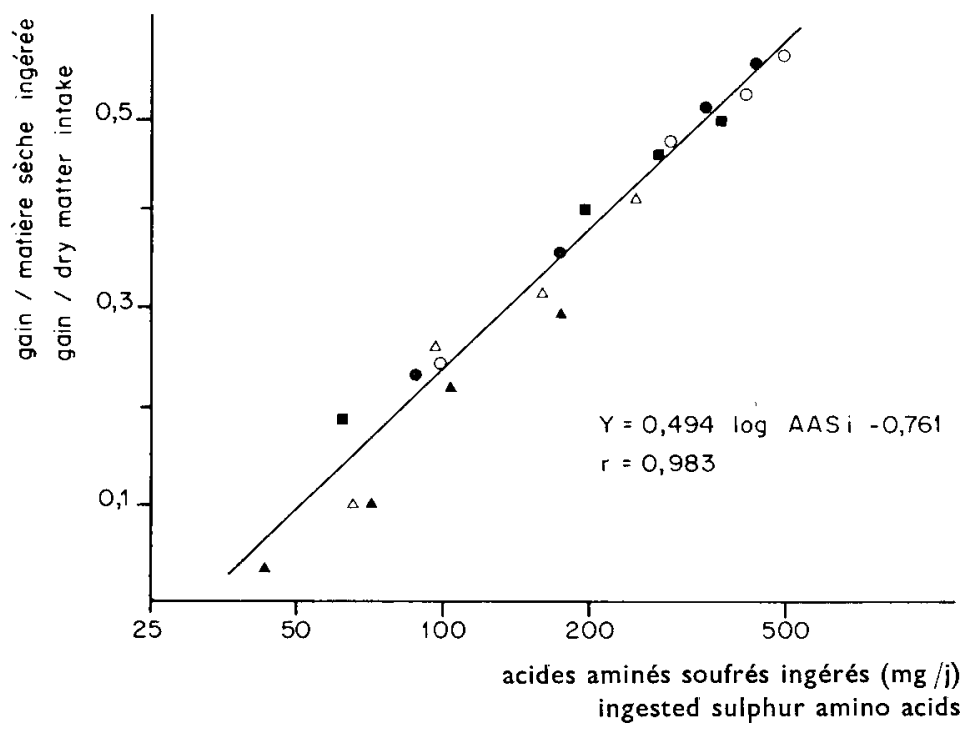

FIG. 2. - Effet de la supplémentation en méthionine de végimes semi-puvifiés à base d'arachide sur

l'efficacité de la conversion alimentaive chez le poulet de chair. Identification des régimes:

Effect of the intake of sulphur amino acids ( $\mathrm{mg} / \mathrm{d}$ ) on teed conversion efficiency (average daily gain/dry matter intake) of semi-purified diets based on peanut meal. Identification of the diets.

Ammoniac (Ammonia treatment) : (O) témoin (control); (•) traité isotourteau (treated iso-peanut

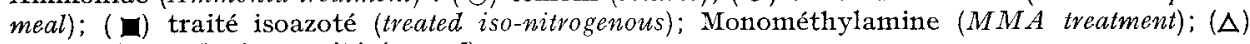
témoin (control); (A) traité (treated). 
transformation partielle en o-coumarine et la non toxicité de cette dernière pour l'œuf embryonné; de la même manière, Doldear et al. (I968), FERrando et al. (I975), chez le caneton, BREKKE et al. (I977) sur l'alevin de truite, prouvent l'efficacité du traitement. Par contre, il est peu vraisemblable, comme le confirment Smith et Hamirton (I978), que, dans des tests courts sur animaux de plus de Io jours, la présence d'aflatoxines puisse jouer un rôle sensible sur les performances de croissance et d'efficacité alimentaire, ce que paraît confirmer le comportement du témoin pauvre en aflatoxine dans le premier essai d'efficacité globale.

La présence de facteurs d'inappétence - ammoniac ou méthylamine - est un aspect à ne pas négliger. Dans un essai de mesure du "Protein Efficiency Ratio ", MANN et al. (I97I) constatent, sans la commenter, une réduction chez le rat de l'ingestion alimentaire $(-8 \mathrm{p}$. roo) de régime à base de tourteau traité à la méthylamine. Pour le porc, JENSEN et al. (I977) n'observent pas d'effet d'inappétence avec le maïs traité à l'ammoniac lorsque le taux de cette substance ne dépasse pas $0, I$ p. Ioo dans le régime présenté en farine, même si la réaction des animaux est différente en consommation en libre choix. Chez la poule pondeuse, WaLdroup et al. (I976) constatent que la substitution poids pour poids de tourteau de coton traité à l'ammoniac au témoin correspondant n'entraîne qu'une réduction marginale de l'efficacité alimentaire, tant que le niveau d'ingestion reste suffisant pour que ne se manifeste pas la carence en acides aminés soufrés que laisse prévoir l'analyse. Aucun critère de qualité de l'œuf n'est modifié. Dans un autre essai de CALVER'T, cité par BREKKE et al. (I 977), l'effet de 1'ammoniation sur l'ingestion alimentaire se marque si le maïs est traité à plus de I p. Ioo d'ammoniac et dose plus de 0,29 p. Ioo d'ammoniac extractible à l'eau, soit 0,2 p. Ioo du régime. Par contre, REID (I 972) signale une amélioration du taux de ponte par traitement à l'ammoniac du tourteau de coton qui ajoute au régime de la pondeuse un taux de $0,3 \mathrm{p}$. I oo d'azote ammoniacal; mais ce même auteur recommande chez le poulet de chair de ne pas dépasser $0, \mathbf{I} 2 \mathrm{p}$. Ioo d'azote ammoniacal, en raison de la toxicité de cette substance pour le jeune animal. Ces doses sont légèrement inférieures à celles incorporées dans nos essais entre 3 et 7 semaines (o, I6 p. roo dans le test d'efficacité globale; 0,23 p. roo dans l'essai de supplémentation en méthionine). Elles n'ont eu qu'un effet négligeable, même au taux le plus élevé, sur l'ingestion alimentaire, et ce, en régime purifié et fortement carencé en acides aminés soufrés. Il n'y a donc pas, sur ce plan, d'inconvénient à l'incorporation de tourteau à moins de 0,6 p. Ioo d'ammoniac dans le régime des jeunes animaux, compte tenu des taux maximum d'incorporation de ces matières premières dans le régime du poulet de chair. La neutralisation des aliments traités par l'acide chlorhydrique a été suggérée par THIESEN (1977) en vue de réduire la forte odeur ammoniacale de produits traités en atmosphère contrôlée, à température ambiante et pression atmosphérique. Elle ne peut agir que sur l'appétence du produit traité. Ces limites ne concernent pas l'alimentation des volailles de ponte, plus tolérantes, ou des ruminants, pour lesquels aucun facteur d'inappétence n'est signalé (ChomyszyN \& ZroLECKA, I972), tant que la température de traitement reste inférieure à Ioo ${ }^{\circ} \mathrm{C}$.

La méthylamine a un effet négatif bien plus marqué et, même à la dose où elle est détectée dans le présent essai sous forme de base azotée volatile dans un extrait à l'acide trichloracétique $(0,06$ p. Ioo dans l'essai d'efficacité globale; o,og p. Ioo dans le test de supplémentation en méthionine), elle paraît exercer un effet négatif marqué sur l'ingestion alimentaire, alors que la dose de traitement prévue $(0,5 \mathrm{p}$. Ioo) et l'enrichissement en azote qui en résulte $(+0,4 \mathrm{p}$. roo) sont nettement plus faibles qu'après le traitement à l'ammoniac. D'après ManN et al. (I97I), l'ingestion de méthylamine serait sans danger. Ces auteurs notaient bien une 
augmentation $\mathrm{d} u$ volume $\mathrm{du}$ foie ainsi que $\mathrm{d} u$ taux de certaines transaminases hépatiques après go jours d'un régime à $25 \mathrm{p}$. Ioo de tourteau de coton détoxifié par ce réactif, mais ces valeurs revenaient à la normale après 5 semaines de retour au régime témoin.

La teneur en cystine est réduite par le traitement. La moindre efficacité des produits traités, d'abord observée par DOLLEAR et al. (I968) et confirmée par MANN et al. (I97I) pour le traitement à l'ammoniac, a peu d'influence chez les animaux pour lesquels les acides aminés soufrés ne sont pas les plus limitants: WaL,droup et al. (1976) le constatent chez la poule pondeuse seulement dans les cas où l'ingéré alimentaire devient insuffisant (stress thermique, par exemple). REID (I972) note, par contre, une amélioration de disponibilité par analyse fécale, que l'on pourrait rapprocher du résultat d'ADRIAN (I976) et de l'amélioration constatée dans notre essai sur un tourteau d'arachide $\left(\mathrm{AO}_{1}\right)$ initialement de qualité médiocre. Mais, c'est surtout chez les espèces les plus exigeantes en acides aminés soufrés que l'effet du traitement est le plus marqué. BELFBEAU, Gousse et WEIL (I974) constatent, chez le rat, sous l'effet du traitement, une réduction du coefficient d'efficacité protéique, tandis que N'GUYEN (I973) observe, chez le poulet de chair, une réduction de l'efficacité biologique du tourteau traité, proportionnelle à son taux d'incorporation au régime.

Il est intéressant de noter enfin que tous les essais d'évaluation biologique ont porté sur des produits traités comparés aux témoins correspondants à même taux d'incorporation dans les farines. Si cette substitution est basée sur la teneur en azote des tourteaux, il en résulte un appauvrissement du régime en acides aminés, déjà signalé par REID (I972) et dont nos essais confirment l'effet négatif sur l'efficacité du régime $d u$ poulet de chair.

Accepté pour publication en décembre 1980.

\section{Remerciements}

Les travaux sur l'ammoniation du tourteau d'arachide ont été effectués dans le cadre d'une action concertée de la D.G.R.S.T. (contrat : 74-7-1056). Le traitement à la méthylamine a été réalisé sous le contrôle de $C$. GIDDEy (BATTELLE, Genève) et le financement de notre recherche d'évaluation assuré par les établissements PETERSEN (Dakar). Qu'ils en soient ici remerciés.

\section{Summary \\ Aflatoxin inactivation by ammonia or monomethylamine treatment Efficiency of treated products in growing chicken}

Gazeous ammonia at a temperature below Ioo ${ }^{\circ} \mathrm{C}$ (VIROBEN et al., I978) or monomethylamine (MMA; GIDDEx, BRANDT et BUNTER, I977) are effective in reducing the level of aflatoxin in contaminated peanut meal to less than $I: 20$ of its initial content (table $I$ ). However, both treatments increase the content of non protein nitrogen and reduce the cystine content of the treated products.

The efficiency of the treatment was evaluated in the chicken using two different diets:

a) maize + peanut at a 22 p. Ioo protein level with added lysine and threonine, thus limited primarily by its content in sulphur amino-acids;

b) a semi-purified diet with peanut meal as sole source of protein + various amino-acids and incremental addition of methionine (o-0.1 - $0.2-0.3 \mathrm{p}$. roo of the diet). 
In the first test, similar to the total protein efficiency test suggested by WoodHaM (I968), the ammonia treatment did not result in any change in feed intake and led to a slight reduction (-3.8 p. IOO) in daily gain and feed conversion efficiency (FCE) between 21 and 49 days. With MMA, tested on younger animals (1o -24 days), the treatment affected feed intake and thus growth and FCE (tabl. 3).

With semi-purified diets, addition of methionine always improved the daily gain and FCE, (tabl. 4 ; fig. $x$ and 2 ). When FCE was plotted against the daily intake of sulphur amino-acids on a logarithmic scale, a high correlation $(r=0.983)$ was obtained. Accordingly there was no decrease in availability of the amino-acids from the treatments under comparison, all the differences being explained by the lower cystine content and the lower acceptance of the treated products. Maximum levels of 0.6 p. roo TCA soluble volatile bases for the ammonia treatment and less than 3 times this level for the MMA treatment were suggested from the results of the present assay and other data from the literature.

\section{Références bibliographiques}

ADrian J., 1976. Évolution de la lysine, méthionine et cystine dans le tourteau d'arachide traité à l'ammoniac. Rev. Fr. Corps Gras, 23, 209-2 I 2.

ANONYME, I972. Recommandations industrielles en alimentation animale. Doc. techn. 111, AEC, Commentry, éd., $4^{\circ} \mathrm{p}$.

BeifebeaU M. J., Gousse R., WEIL A., I974. Valeur nutritionnelle pour le rat en croissance d'un tourteau d'arachide détoxifié par ammoniation. Rev. Fr. Corps Gras, 21, 469-473.

BREKKF, O. L., PEPLiNski A. J., LaNCASTER E. B., 1977. Aflatoxin inactivation in corn by aqua ammonia. Trans. A.S.A.E., 20, I I60.

Brekke O. I., Sinnhuber R. O., Peplinski A. J., Wales J. H., PutNam G. B., I,ee D. J., CIEGLER A., r977. Aflatoxin in corn : ammonia inactivation and bioassay with rainbow trout. Applied and Envir. Microbiol., 34, 34-37.

CHOMYSZYN M., ZIOLECKA A., 1972. Utilization of ammoniated feeds in ruminant nutrition in "Tracer studies on non protein nitrogen for ruminants". International Atomic Enevgy A gency, Vienna, I53-16r.

Combs G. F., Bossard E. H., Childs G. R., 1968. Improved chick bioassays for available lysine and available methionine. Feedstuffs, Feb. 24, 36-38.

CUCUI, IU A. F., LEE L. S., PONS W. A. Jr, STANL, J. B., 1976. Ammoniation of aflatoxin $B_{1}$ : isolation and characterization of a product with molecular weight 206. J. Agric. Food Chem., 24, 408-410.

DoLIEAR F. G., I969. Detoxification of aflatoxins in foods and feeds in Goldblatt L.A. " $A$ flatoxin. Scientific background, control and implications". Acad. Press, New York, 359-39r.

Dolifar F. G., Mann G. E., Codifer I. P. Jr, Gardner H. K. Jr, Koltun S. P., Vix H. I. E., 1968. Elimination of aflatoxins from peanut meal. J. Amer. Oil Chem. Soc., 45, 862-865.

Ferrando R., Parodi A. L., Henry N., N'Diaye A. I., Fourlon Cl., Delort-Laval J., I975. Influence d'un traitement par l'ammoniaque du tourteau d'arachide contaminé par $A$. flavus sur son efficacité alimentaire chez le caneton. Ann. Nutr. Alim., 29, 6I-66.

Giddey C., Brandt J., Bunter G., 1977. The detoxification of oil-seed cakes polluted by aflatoxins. Research and development of an industrial process. Ann. Technol. Agric., 27, 331338 .

Gupta J. D., Dakroury A. M., Harper A. E., Elvehjem C. A., I958. Biological availability of lysine. J. Nutr., 64, 259-270.

HARwoOd E. J., SHRImpton D. H., I969. A chick biological assay for available methionine. Proc. Nutr. Soc., 28, 67A-68A.

Jensen A. H., Brekke O. L., Frank G. R., Peplinski A. J., I977. Acceptance and utilization by swine of aflatoxin-contaminated corn treated with aqueous or gaseous ammonia. J. Anim. Sci., 45, 8-I2.

IEE L. S., Stanley J. B., Cucullu A. F., Pons W. A. Jr, Goldbiamt L. A., i974. Ammoniation of aflatoxin $\mathrm{B}_{1}$ : isolation and identification of the major reaction product. $J$. Assoc. Off. Anal. Chem., 57, 626-63r.

Mann G. E., Gardner H. K. Jr, Booth A. N., Gumbmann M. R., I97I. Aflatoxin inactivation. Chemical and biological properties of ammonia and methylamine treated cottonsead meal. J. Agvic. Food Chem., 19,. I $155^{-\mathrm{I}} 5^{8}$. 
MCGHEE J. E., BAGLey E. B., Bothast J. R., I979. Treating corn with aqua ammonia: effect on meal constituents. Cereal Chem., 56, I28-129.

Millier E. L., Carpenter K. J., Morgan C. B., BovNe A. W., ig65. Availability of sulphur amino acids in protein foods. 2 . Assessment of available methionine by chick and microbiological assays. Brit. J. Nutr., 19, 249-267.

MOREAU C., 1976. Variations de la pollution fongique des arachides et de leurs tourteaux de la récolte à la consommation. Rev. mycologie, 40, 97-I 15.

N'GUyEN, 1973. In Travaux concernant l'aflatoxine. Extrait du rapport d'activité laboratoire, Institut des Corps Gras, Paris, 60-63.

REID R. L., I972. Ammoniated cottonseed meal in poultry diets. Feedstuffs, Nov. 6, 42.

Suiru J. W., Hamition P. B., I97o. Aflatoxicosis in the broiler chicken. Poult. Sci., 49, 207-215.

Szylit O., Delort-Laval J., Borgida L. P., i974. Dégradation dans le jabot du coq et efficacité d'amidons de maïs à différents taux d'amylose sur la croissance du poulet. Ann. Zootech., 23, 253-265.

THIESEN J., 1977. Detoxification of aflatoxins in groundnut meal. Anim. Feed Sci. Technol, 2, $67-75$.

Vesonder R. F., Beckwith A. C., Ciegler A., Dimler R. J., 1975. Ammonium hydroxyde treatment of aflatoxin $\mathrm{B}_{1}$. Some chemical characteristics and biological effects. J. Agric. Food Chem., 23, 242-243.

Viroben G., DelorT-Laval, J., Colin J., Adrian J., i978. Inactivation des aflatoxines pat traitement à l'ammoniac. Études in vitro de tourteaux d'arachides détoxiqués. Ann. Nutr. Alim., 32, 167-1 85 .

Waldroup P. W., Hazen K. R., Mitchell R. J., Payne J. R., Johnson Z., 1976. Ammoniated cottonseed meal as a protein supplement for laying hens. Poult. Sci., 55, ror r-ror 9.

WoOdHAm A. A., I968. A chick growth test for the evaluation of protein quality in cerealbased diets. Brit. Poult. Soi, 9, 53-63. 